\title{
Exergy Analysis of a Compartment Fire
}

\author{
V. Bertola ${ }^{1}$ and E. Cafaro ${ }^{2, *}$ \\ ${ }^{I}$ I.S.I. Foundation, Viale Settimio Severo 65, 10133 Torino, Italy \\ ${ }^{2}$ Dipartimento di Energetica, Politecnico di Torino, Corso Duca degli Abruzzi 24, 10129 Torino, Italy
}

\begin{abstract}
A thermodynamic model of a compartment fire with one state variable (corresponding to the dimensionless temperature of the smoke layer) based on energy conservation is studied from the point of view of the Second Law (exergy analysis). To define the exergy rate of the process, a novel concept of mean thermodynamic temperature is introduced for the smoke layer. This parameter is the equivalent of the Carnot factor in the energy transfer process for an open system under steady-state conditions. The exergy analysis shows that in order to satisfy the Second Law, not all the solutions of the thermodynamic model are physically accessible in the phase space. The physically accessible part of the phase space is delimited by a boundary representing the non-negative entropy generation condition.
\end{abstract}

Keywords: Compartment fire, semenov model, exergy analysis.

\section{INTRODUCTION}

The study of compartment fires is an important area of fire safety engineering, which has received much attention in the past few decades, and several models were developed in order to understand the fundamental mechanisms of fire growth and to assess fire hazard [1,2]. Deterministic models can be sorted into zone models, which allow one to find the main parameters of the fire by solving a set of first-order ordinary differential equations derived from global balance equations supplemented by semi-empirical physical models [3], and field models, which simulate the fire evolution within extended geometric domains by solving a set of discretised partial differential equations using general-purpose CFD packages where specific sub-models have been introduced to describe the effects of buoyancy, radiation heat transfer and turbulence [4]. Stochastic approaches view the fire growth as a percolation process, where the transition from non-propagating to propagating fire is described as a phase change phenomenon [5].

One of the main objectives of such models is to predict whether a fire will extinguish spontaneously or grow until it reaches a point when the flame spreads almost instantaneously to occupy the whole enclosure, which is known as flashover [6]. However, even a qualitative description of the phenomenon is not easy to obtain, both because of the complexity of the physical model, which must take into account the radiative heat exchange between the flame, the fuel, and the surroundings, and because most of the physical and environmental parameters (such as the burning surface and the ventilation conditions) are variable in time in a way that is generally not predictable a priori. Flash-over and extinction jumps [6-9] as well as the hysteresis between the fuelcontrolled and ventilation-controlled regimes [10] have been

\footnotetext{
*Address correspondence to this author at the Politecnico di Torino, Corso Duca degli Abruzzi 24, 10129 Torino, Italy; Tel: +39 011 5644476; Fax: +39011 5644499; E-mail: emilio.cafaro@polito.it
}

experimentally observed and recast in mathematical models. A number of studies aimed at understanding other parametric effects, such as the effect of the thermal inertia of the walls [11], of the discharge coefficient [12], and of the aspect ratio of the compartment [13]. In particular, mathematical models with one $[9,11,14,15]$, two [8] or three state variables [12, $13,16]$ are available in the literature. More recently, the effects of stochastic fluctuations of the model variables have been investigated $[17,18]$. In particular, it has been shown that while purely Gaussian perturbations (white noise) do not change the behaviour of the fire with respect to the deterministic model, perturbations depending on the model variable ('coloured' noise) may drive the system to different states [18].

This work aims to get a deeper understanding of the behaviour of compartment fires and their stability by means of the exergy analysis of a one-variable thermodynamic model for compartment fires, conceptually analogous to Semenov's theory of thermal ignition. After establishing an analogy between the compartment fire and a virtual heat exchanger, which transfers thermal energy from the compartment to the surrounding environment, the fire behaviour can be characterised by the exergetic number (i.e., the non-dimensional exergy) exchanged or destroyed in the process.

Unlike other similar approaches based on Semenov's energy conservation model, the exergy analysis allows one to take into account the Second Law of thermodynamics, i.e. the non-negative entropy generation condition, which introduces an additional constraint on the model solutions.

\section{THERMODYNAMIC MODEL OF COMPART- MENT FIRE}

The simplest approach to describe fires in enclosures is the zone model with pool fire combustion, which is schematically represented in Fig. (1). Within this framework, one can build a thermodynamic model of the fire dynamics where, like in Semenov's theory of thermal explosion, the net rate of increase of the energy of the upper layer (i.e., the 


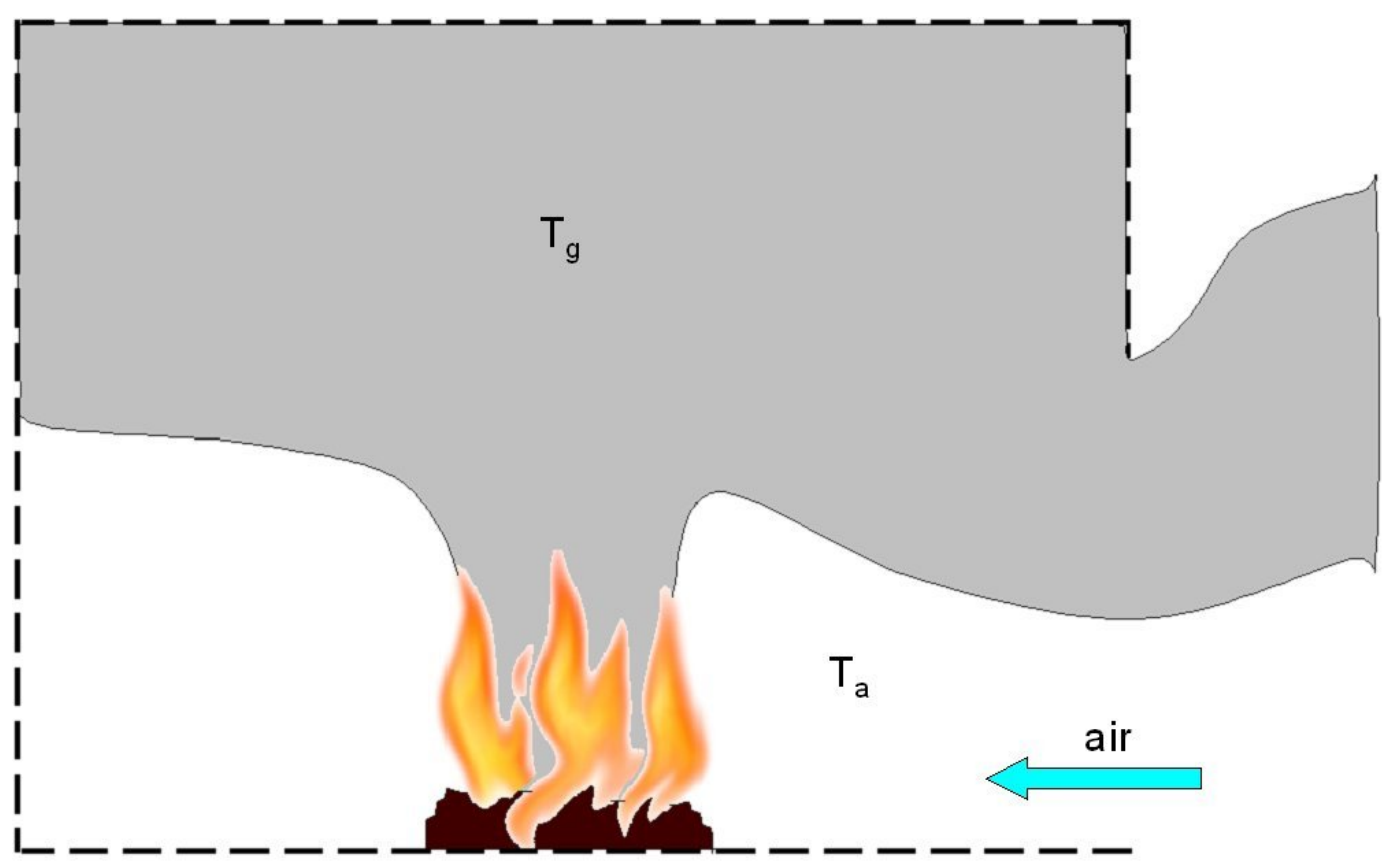

Fig. (1).

combustion products) is given by the difference between the combustion heat transferred to the smoke layer and that rejected to the surroundings $[15,18]$.

The total energy produced by combustion is $\eta_{c} \dot{m}_{f} H_{c}$, where $\eta_{\mathrm{c}}$ is the combustion efficiency, $\dot{m}_{f}$ the mass flow rate of fuel, and $H_{c}$ the lower heat of combustion, while the fraction of such energy that is actually transferred to the smoke is given by [19]:

$\mu=1-\exp \left[-\frac{\dot{m}_{a} / \dot{m}_{f}}{\left(\dot{m}_{a} / \dot{m}_{f}\right)_{s}}\right]$

where $\dot{m}_{a}$ is the mass flow rate of fresh air and the subscript $\mathrm{s}$ denotes stoichometric conditions. The mass flow rate of fuel that sustains combustion can be obtained from an energy balance between the vaporization heat of the solid or liquid fuel, $\Delta H_{\text {vap }}$, and the radiation heat rate on its surface, $A_{f}$, assuming that the heat source for the fuel vaporization is radiation from the smoke layer and the flame itself [20]:

$\dot{m}_{f}=\frac{A_{f} \dot{q}_{r}}{\Delta H_{v a p}}$

where $\dot{q}_{r}$ is the radiative heat flux, which depends on the temperatures of the flame, of the exhaust gas and of the combustion surface, as well as on their shape factor and emissivity [18], and can be written in the form:

$\dot{q}_{r}=\sigma\left(\gamma T_{a}^{4}+\varepsilon T_{g}^{4}\right)$

where $\sigma$ is the Stefan-Boltzmann constant, the coefficient $\varepsilon$ accounts for the radiative properties of the gas, and $\gamma$ for all the other variables. The explicit representation of radiative heat transfer from the flame in the context of this model can be found in Ref. [18]. For simplicity, the assumption $\varepsilon=1$ will be used henceforth.
The argument of the exponential in Eq. (1) is the inverse of the equivalence ratio, and is used to model the effect of ventilation conditions on the combustion efficiency for any kind of fuel. Finally, the energy generation rate can be expressed in the form:

$\dot{G}\left(T_{g}, t\right)=\mu \eta_{c} \dot{m}_{f} H_{c}$

and obviously depends on the smoke temperature, $T_{g}$, and on time.

As for the thermal energy transferred to the surrounding environment, this is partly carried by the exhaust gas stream and partly transferred by convection to the compartment walls, so that it can be written as:

$\dot{R}\left(T_{g}, t\right)=\dot{m}_{g} c_{g}\left(T_{g}-T_{a}\right)+h A\left(T_{g}-T_{a}\right)$

where $h$ is the overall heat transfer coefficient and $A$ the effective heat transfer surface. The energy equation can be written as:

$\rho_{g} V_{g} c_{g} \frac{d T_{g}}{d t}=\mu \eta_{c} \dot{m}_{f} H_{c}-\left[\dot{m}_{g} c_{g}\left(T_{g}-T_{a}\right)+h A\left(T_{g}-T_{a}\right)\right]$

where $\dot{m}_{g}=\dot{m}_{a}+\dot{m}_{f} \approx \dot{m}_{a}$, and $\rho_{g}$ and $V_{g}$ are the smoke density and the compartment volume, respectively. A detailed analysis of the model described by Eq. (6) is reported elsewhere $[15,18]$. Here, the analysis is limited to the steady state:

$\frac{\mu \eta_{c} \dot{m}_{f} H_{c}}{h A}-\left[\frac{\dot{m}_{g} c_{g}}{h A}\left(T_{g}-T_{a}\right)+\left(T_{g}-T_{a}\right)\right]=0$

The energy balance can be re-written in dimensionless form after identifying the following parameters:

$\theta=\frac{T_{g}-T_{a}}{T_{a}}$ 
$N_{S}=\frac{h A}{\dot{m}_{g} c_{g}}$

$\alpha=\frac{A_{f} \sigma T_{a}^{3} \eta_{c} H_{c}}{h A \Delta H_{v a p}}$

$\lambda=\frac{c_{g} T_{a}\left(\dot{m}_{a} / \dot{m}_{f}\right)_{s}}{\eta_{c} H_{c}}$

so that Eq. (7) becomes:

$\alpha\left[\gamma+(1+\theta)^{4}\right]\left[1-\exp \left(-\frac{1}{\alpha \lambda N_{S}\left[\gamma+(1+\theta)^{4}\right]}\right)\right]-$

$\frac{1+N_{S}}{N_{S}} \theta=0$

Fig. (2) shows some examples of solutions of Eq. (12) with respect to the dimensionless temperature, for different values of the parameters. The problem can have one or three solutions (one of which unstable), which can be interpreted in terms of flashover (high temperature branches) or extinction (low temperature branches).
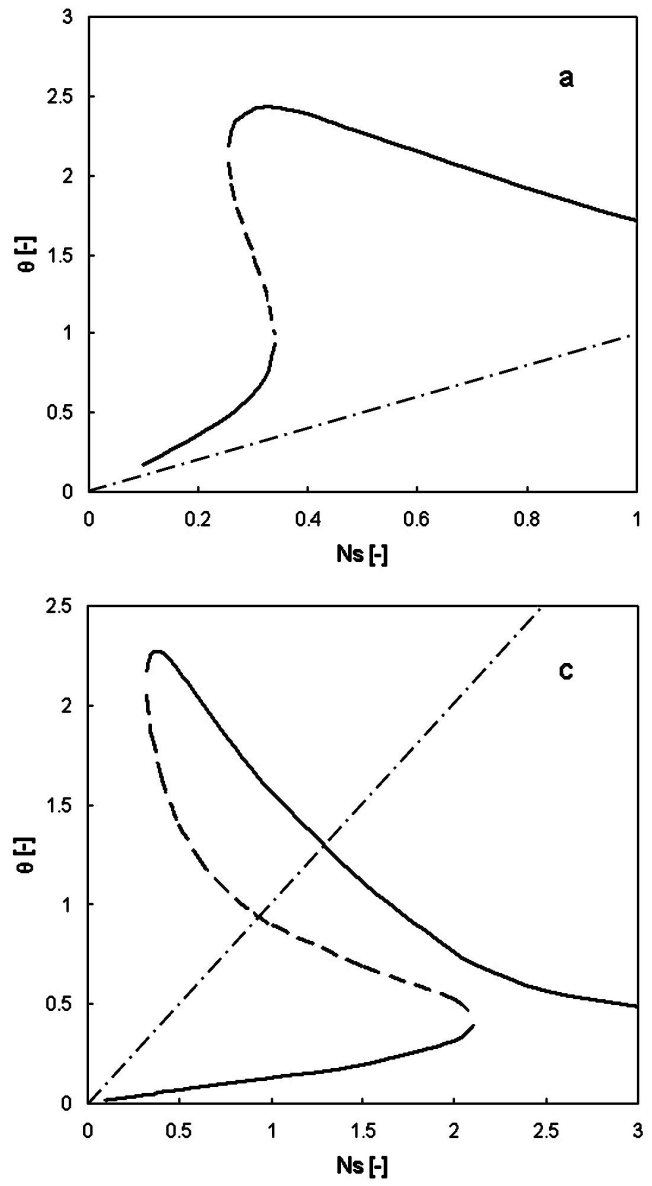

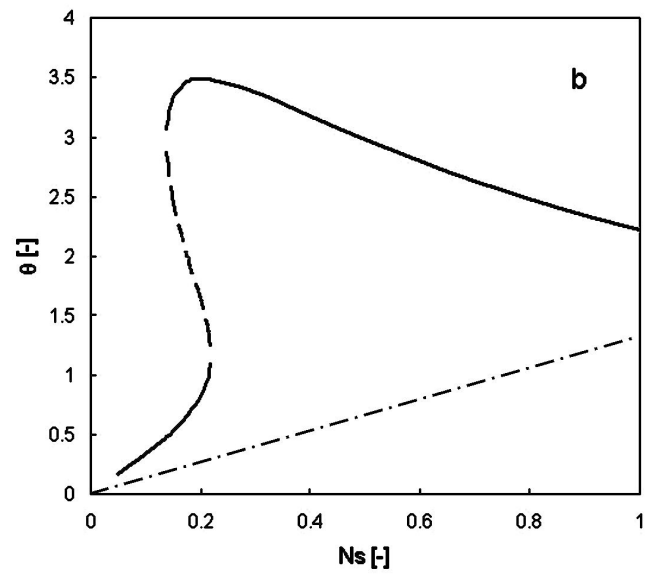

\section{EXERGY ANALYSIS}

The exergy balance equation for the smoke layer is given by $[21,22]$ :

$\dot{B}_{g}=\dot{H}_{g}-T_{a} \dot{S}_{g}$

where, under the assumption of ideal gas and neglecting pressure drops, the total entropy rate can be written as:

$\dot{S}_{g}=\dot{m}_{g} c_{g} \ln \frac{T_{g}}{T_{a}}$

and the rate of total enthalpy is:

$\dot{H}_{g}=\dot{m}_{g} c_{g}\left(T_{g}-T_{a}\right)$

The exergy balance can be re-written in terms of the dimensionless temperature as:

$\dot{B}_{g}=\dot{H}_{g}\left[1-T_{a} \frac{\Delta S_{g}}{\Delta H_{g}}\right]$

where $\Delta H_{g}$ and $\Delta S_{g}$ represent the enthealpy and entropy variation of the smoke layer, respectively. Recalling Eqs. (14) and (15) one can also write:

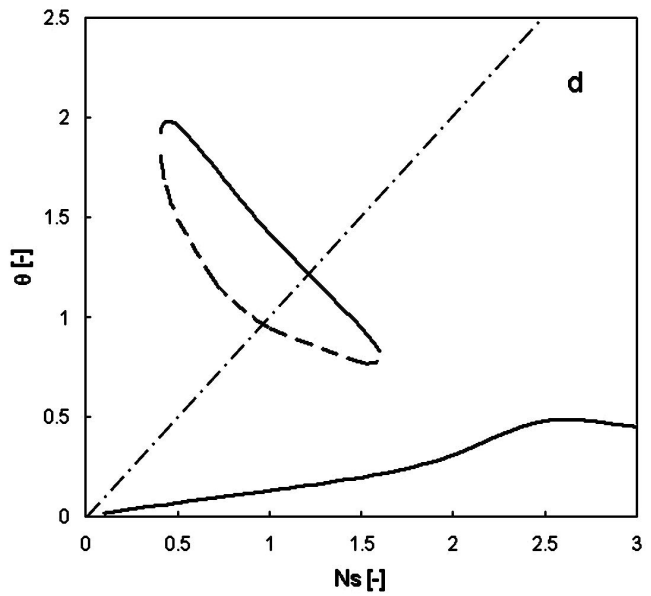

Fig. (2). Equilibrium solutions of the energy conservation model of a compartment fire, for different values of the parameters: (a) $\alpha=0.16, \lambda$ $=0.2, \gamma=10 ;(\mathbf{b}) \alpha=0.16, \lambda=0.2, \gamma=20 ;(\mathbf{c}) \alpha=0.16, \lambda=0.24, \gamma=0 ;(\mathbf{d}) \alpha=0.16, \lambda=0.25, \gamma=0$. Solid lines correspond to stable solutions (extinction or flashover), while broken lines correspond to unstable solution branches. The dash-dot line represents the thermodynamic limit based on Eq. (27). 


$$
\dot{B}_{g}=\dot{H}_{g}\left[1-\frac{\ln (\theta+1)}{\theta}\right]
$$

The ratio between the exergy and enthalpy variations of the gas defines the exergetic number:

$N_{E}=1-T_{a} \frac{\Delta S_{g}}{\Delta H_{g}}$

The parameter defined by Eq. (18) is the equivalent of the Carnot factor in the energy transfer process for an open system under steady-state conditions. In particular, the ratio $\Delta H_{g} / \Delta S_{g}$ corresponds to the actual mean thermodynamic temperature of the heat transfer process under consideration:

$$
T_{m}=\frac{\Delta H_{g}}{\Delta S_{g}}=\frac{T_{g}-T_{a}}{\ln \frac{T_{g}}{T_{a}}}
$$

Assuming that the wall temperature is constant, and that pressure drops within the control volume can be neglected, the thermodynamic system represented by the smoke layer can be described as a fictitious heat exchanger, characterized by a uniform distribution of one of the process fluids. The rate of enthalpy of the hot gas can therefore be written in the form:

$$
\dot{H}_{g}=h A T_{m}
$$

where $T_{m}$ is the mean thermodynamic temperature defined above in Eq. (19). Note that this definition of temperature is different from the conventional definition of logarithmic mean temperature adopted in the heat exchanger theory. The two temperatures become coincident only if the cold side of the fictitious heat exchanger is an ideal heat reservoir at the absolute zero.

The comparison between Eqs. (15) and (20) allows one to write:

$\ln \frac{T_{g}}{T_{a}}=\frac{h A}{\dot{m}_{g} c_{g}}$

or, in dimensionless form:

$\ln (\theta+1)=N_{S}$

Finally, the identities given by Eqs. (21) and (22) also imply a relationship with the equivalence ratio introduced in Eq. (1):

$\ln (\theta+1)=\frac{1}{\alpha \lambda\left[\gamma+(1+\theta)^{4}\right]} \frac{\dot{m}_{a} / \dot{m}_{f}}{\left(\dot{m}_{a} / \dot{m}_{f}\right)_{s}}$

Having established the correct expression of the mean thermodynamic temperature of the energy transfer process from the smoke layer to the environment, one can write the exergy balance for the full transient model, where the total enthalpy rate can be written as:

$\dot{H}_{g}=\rho_{g} V_{g} c_{g} \frac{d T_{g}}{d t}$

Recalling Eqs. (6), (16), (17) and (18) yields:
$\dot{B}_{g}=N_{E}\left\{\mu \eta_{c} \dot{m}_{f} H_{c}-\left[\dot{m}_{g} c_{g}\left(T_{g}-T_{a}\right)+h A\left(T_{g}-T_{a}\right)\right]\right\}$

and finally one obtains the dimensionless form:

$$
\frac{\dot{B}_{g}}{h A T_{a}}=N_{E}\left\{\alpha\left[\gamma+(1+\theta)^{4}\right]\left[1-\exp \left(-\frac{1}{\alpha \lambda N_{S}\left[\gamma+(1+\theta)^{4}\right]}\right)\right]-\frac{1+N_{S}}{N_{S}} \theta\right\}
$$

The comparison between Eq. (17) and Eq. (22), together with the definition of number of exergy units (Eq. 18), gives a relationship between $\mathrm{N}_{\mathrm{E}}$ and $\mathrm{N}_{\mathrm{S}}$ :

$N_{S}=\theta\left(1-N_{E}\right)$

The relationship given in Eq. (27) allows one to use the number of exergy units as a control parameter for the fire evolution model instead of the parameter $N_{S}$. Introducing Eq. (27) into Eq. (26) yields:

$$
\left.\frac{\dot{B}_{g}}{h A T_{a}}=N_{E}\left\{\alpha\left[\gamma+(1+\theta)^{4}\right]\right]\left[-\exp \left(-\frac{1}{\alpha \lambda \theta\left(1-N_{E}\right)\left[\gamma+(1+\theta)^{4}\right]}\right)\right]-\frac{1+\theta\left(1-N_{E}\right)}{1-N_{E}}\right\}
$$

Examples of dimensionless exergy plots calculated from Eq. (28) are shown in Fig. (3).

Because by definition $0 \leq N_{E} \leq 1$, Eq. (27) represents a thermodynamic limit for the occurrence of fire evolution: in particular, we have that $N_{S} \leq \theta$, which is equivalent to $\ln (\theta+1) \leq \theta$. As a consequence, not all the equilibrium solutions of the model that satisfy conservation of energy, i.e. solutions of Eq. (12), are also physically admissible. The line describing the thermodynamic limit is plotted on the graphs of equilibrium solutions reported in Fig. (2), for different values of the model parameters. While the solutions of the energy conservation model in Figs. (2-a) and (2-b) are all admissible, in Figs. (2-c) and (2-d) only a part of the highertemperature branch (corresponding to flashover) can occur in practice.

\section{CONCLUSIONS}

The dynamics of compartment fires can be described through a generalized Semenov model with one state variable, based on the balance between the rate of heat generation and the energy transferred to the surrounding environment. The equilibrium solutions of the model can be interpreted in terms of extinction or flashover.

Based on the energy conservation model, one can build up the exergy analysis of the system. In particular, it is possible to put exergy in a dimensionless form by introducing the exergetic number, defined as the ratio between the exergy and the enthalpy of the smoke layer. This number is the equivalent of the Carnot factor in the energy transfer process for an open system under steady-state conditions. In this context, the ratio between the enthalpy and the entropy variation of the smoke layer corresponds to the actual mean thermodynamic temperature of the heat transfer process between the fire and the surrounding environment.

Because the exergy number must be positive and smaller than unity, the exergy analysis introduces an additional constraint on the steady-state solutions of the thermodynamic 

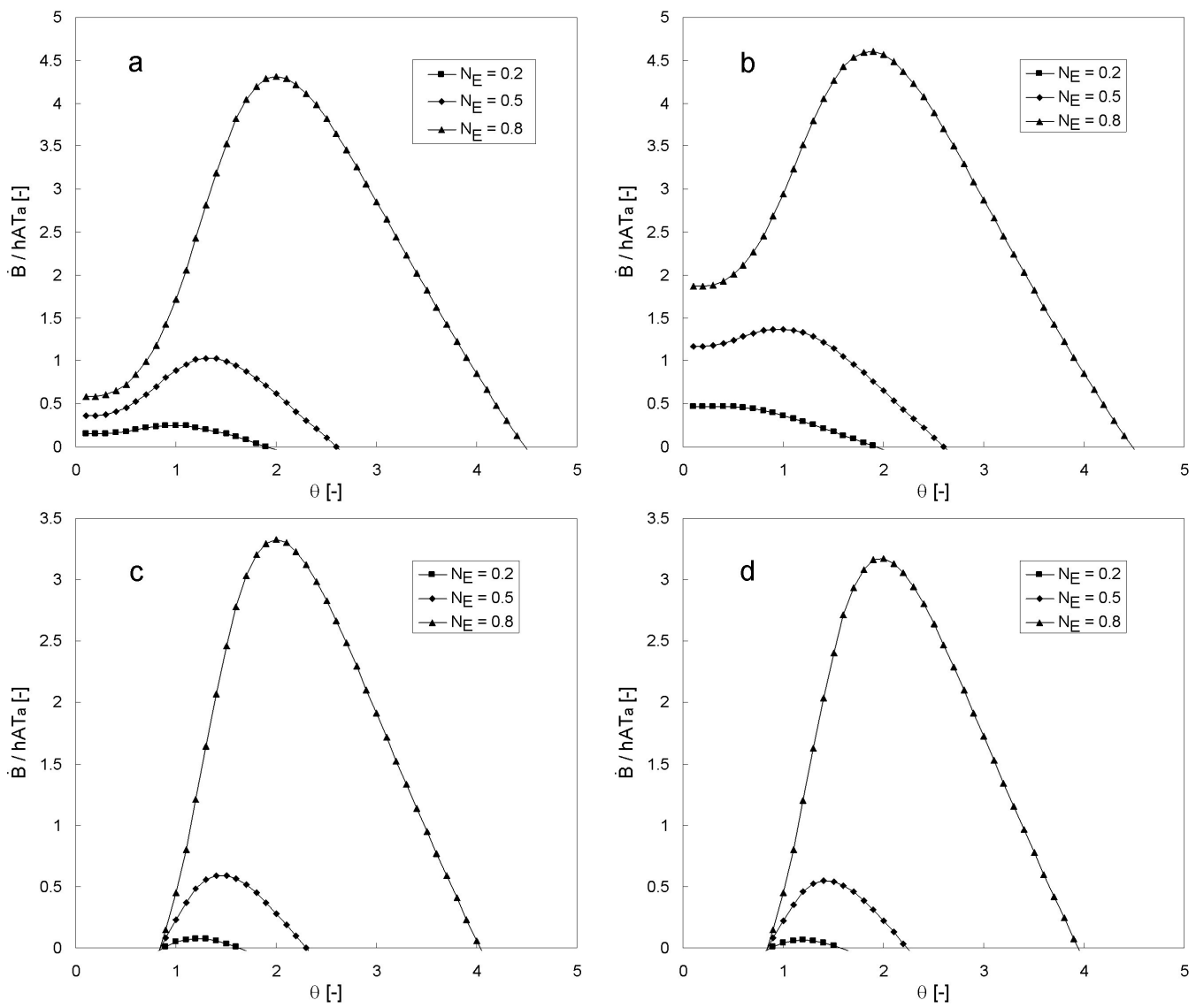

Fig. (3). Dimensionless exergy of the smoke layer calculated from Eq. (28): (a) $\alpha=0.16, \lambda=0.2, \gamma=10$; (b) $\alpha=0.16, \lambda=0.2, \gamma=20$; (c) $\alpha$ $=0.16, \lambda=0.24, \gamma=0 ;$ (d) $\alpha=0.16, \lambda=0.25, \gamma=0$.

model based on the energy balance: thus, not all of these solutions are physically admissible.

\section{ACKNOWLEDGEMENTS}

This work was partly supported by the Royal Society through an International Joint Project grant (JP0867566 2008/R1).

\section{NOMENCLATURE}

$$
\begin{array}{ll}
\mathrm{A} & =\text { Area } \\
\mathrm{B} & =\text { Exergy } \\
\mathrm{c} & =\text { Heat capacity } \\
\mathrm{h} & =\text { Heat transfer coefficient } \\
\mathrm{H} & =\text { Enthalpy } \\
\mathrm{H}_{\mathrm{c}} & =\text { Heat of combustion } \\
\dot{m} & =\text { Mass flow rate } \\
\mathrm{N}_{\mathrm{E}} & =\text { Exergetic number } \\
\mathrm{N}_{\mathrm{S}} & =\text { Ventilation number } \\
\mathrm{q} & =\text { Heat flux } \\
\mathrm{t} & =\text { Time } \\
\mathrm{S} & =\text { Entropy } \\
\mathrm{T} & =\text { Temperature }
\end{array}
$$

$\mathrm{V}=$ Compartment volume

\section{Greek symbols}

$\alpha=$ Dimensionless heat of combustion

$\gamma=$ Radiative properties of flame and combustion surface coefficient

$\varepsilon=$ Radiative properties of the gas coefficient

$\eta \quad=$ Efficiency

$\lambda=$ Dimensionless energy of fresh air

$\mu=$ Fraction of fuel energy transferred to smoke

$\rho \quad=$ Density

$\sigma \quad=$ Stefan-Boltzmann constant

$\theta=$ Dimensionless temperature

\section{Subscripts}

$\begin{array}{ll}\mathrm{a} & =\text { Air, ambient } \\ \mathrm{c} & =\text { combustion } \\ \mathrm{g} & =\text { Hot gas }\end{array}$

\section{REFERENCES}

[1] D.B. Birk, An introduction to mathematical fire modelling, Basel: Techomic Publishing Company Inc., 1991.

[2] D.D. Drysdale, An Introduction To Fire Dynamics, $2^{\text {nd }}$ Ed. Chicester: Wiley, 1998. 
[3] H.E. Mitler, "The Harvard fire model", Fire Saf. J., vol. 9, pp. 7$16,1985$.

[4] N.C. Markatos, and G. Cox, "Hydrodynamics and heat transfer in enclosures containing a fire source", Physico. Chem. Hydrodynam., vol. 5, pp. 57-66, January 1984.

[5] T. Beer, "Percolation and fire spread", Combus. Sci. Technol., vol. 72, pp. 297-304, November 1990.

[6] P.H. Thomas, M.L. Bullen, J.G. Quintiere, and B.J. McCaffrey, "Flashover and instabilities in fire behavior", Combust. Flame., vol. 38, pp. 159-171, June 1980.

[7] S.R. Bishop, P.G. Holborn, A.N. Beard, D.D. Drysdale, "Dynamic modelling of building fires", Appl. Math. Modell., vol. 17, pp. 170183, April 1993.

[8] S.R. Bishop, P.G. Holborn, A.N. Beard, D.D. Drysdale, "Nonlinear dynamics of flashover in compartment fires", Fire Saf. J., vol. 21, pp. 11-45, March 1993.

[9] T.L. Graham, G. Makhviladze, and J. Roberts, "On the theory of flashover development”, Fire Saf. J., vol. 25, pp. 229-259, October 1995.

[10] P.G. Holborn, S.R. Bishop, D.D. Drysdale, and A.N. Beard, "The effect of variation of ventilation and other control parameters in a compartment fire model", Int. Comm. Heat Mass Transfer, vol. 19, pp. 711-719, October 1992.

[11] T.L. Graham, G. Makhviladze, and J. Roberts, "The Effects of the thermal inertia of the walls upon flashover development", Fire Saf. J., vol. 32, pp. 35-60, February 1999.

[12] A.N. Beard, "Dependence of flashover on assumed value of the discharge coefficient", Fire Saf. J., vol. 36, pp. 25-36, February 2001.
[13] A.N. Beard, "Dependence of flashover on temperature and aspect ratio of the compartment", J. Fire Sci., vol. 21, pp. 267-283, July 2003.

[14] A.N. Beard, D.D. Drysdale, P.Holborn, and S. Bishop, "A nonlinear model of flashover", Fire Sci. Technol., vol. 12, pp. 11-27, November 1992

[15] E. Cafaro, and L. Ranaboldo, "A phase diagram for fires", in Eurotherm Seminar No. 53 "Advanced Concepts and Techniques in Thermal Modelling 2", Mons, Belgium (eds J. Henriette, P. Lybaert \& M. El Hayeck). Paris: Elsevier, 1997.

[16] A.N. Beard, D. Drysdale, P. Holborn, and S. Bishop, "A Model of instability and flashover", J. Appl. Fire Sci., vol. 4, pp. 3-16, 199495.

[17] A. Hasofer, and V. Beck, "A stochastic model for compartment fires”, Fire Saf. J., vol. 28, pp. 207-225, April 1997.

[18] V. Bertola, E. Cafaro, "Deterministic-stochastic approach to compartment fire modelling", Proc. Royal Soci. A., vol. 465, pp. 10291041, April 2009.

[19] A. Tewarson, F.H. Jiang, and T. Morikawa, "Ventilation controlled combustion of polymers", Combust. Flame., vol. 95, pp. 151-169, October 1993.

[20] S.B. Dorofeev, A.A. Efimenko, A.S. Kochurko, and M.S. Kuznetsov, "Industrial fuel ignition conditions by flame radiation", J. Loss Prevent. Proc. Ind., vol. 6, pp. 159-163, May 1993.

[21] A. Bejan, Advanced Engineering Thermodynamics, $2^{\text {nd }}$ Ed. New York: Wiley, 1997.

[22] I. Dincer, and M.A. Rosen, Exergy: Energy, Environment and Sustainable Development, New York: Elsevier, 2007.

(C) Bertola and Cafaro; Licensee Bentham Open.

This is an open access article licensed under the terms of the Creative Commons Attribution Non-Commercial License (http://creativecommons.org/licenses/ by-nc/3.0/) which permits unrestricted, non-commercial use, distribution and reproduction in any medium, provided the work is properly cited. 